\section{New roles for old factor}

\section{By Kai-Jye Lou, Staff Writer}

Researchers at the University Medical Center Utrecht and Crossbeta Biosciences B.V. have linked high levels of activated Factor XII with amyloidosis, a metabolic disease that is characterized by misfolded protein aggregates. ${ }^{1}$ The company plans to use the discovery in two ways-developing diagnostics for amyloidosis and other protein-misfolding disorders and offering services to protein therapeutic developers to measure levels of potentially immunogenic misfolded proteins in their production batches.

Other companies and academics want to see additional mechanistic data demonstrating the activation of Factor XII by misfolded protein aggregates. If the association holds up, they said it could have applications in developing treatments for inflammatory diseases.

Factor XII can be activated by contact with a variety of negatively charged artificial or biological surfaces. Activated Factor XII is involved in both coagulation and inflammation. The protein can initiate the coagulation cascade by cleaving Factor XI, or it can trigger an inflammatory response by activating the kallikrein-kinin and complement systems. ${ }^{1}$

In a paper in The Journal of Clinical Investigation, a research group led by Martijn Geb-

bink, CSO and cofounder of Crossbeta and an associate professor in the Department of Clinical Chemistry and Hematology at University Medical Center Utrecht, showed that plasma samples from patients with systemic amyloidosis had higher levels of activated Factor XII than samples from healthy controls. In vitro assays showed that, in the presence of misfolded proteins, activated Factor XII generates kallikrein from prekallikrein and initiates an inflammation cascade.

The activated Factor XII did not trigger a coagulation cascade. This was welcome news, as there had been concerns that developing strategies to clear misfolded proteins from the blood could impair normal clotting.

Misfolded proteins are found in the damaged tissue of healthy individuals-for example, in tissue injured by a small cut or scrape. As a result, Gebbink's group was worried that by removing misfolded proteins, they might be removing an important component of normal clot formation.

Clive Wood, CSO and EVP of discovery research at Dyax Corp., said the JCI article provides insight on additional pathways that activate plasma kallikrein. "This paper helps to underline that there are other diseases that involve activation of the kallikrein pathway" in addition to those Dyax is currently focusing on, he said.

Currently, plasma kallikrein is known to be activated through autoactivation of Factor XII or through a serine protease present in endothelial cells. ${ }^{2}$ Diseases known to involve activation of the kallikrein pathway include rheumatoid arthritis, Crohn's Disease and hereditary angioedema (HAE).

Dyax's DX-88 ecallantide has completed a second Phase III trial in HAE. The company completed submission of a rolling BLA for the yeast-derived recombinant plasma kallikrein inhibitor on Sept. 24.

Robert Colman, professor of medicine in the Thrombosis Research Center at Temple University, noted that the data in the JCI paper came from patient samples. He told SciBX that Gebbink's group "still needs to demonstrate that it is indeed misfolded proteins that are activating Factor XII in vivo."

Alvin Schmaier, chief of hematology and oncology at Case Western Reserve University, agreed. He said more work is needed to demonstrate that activated Factor XII triggers inflammation but not coagulation in the presence of misfolded proteins.

"In plasma, there is a lot more prekallikrein than Factor XI," Schmaier noted. As a result, he suggested Factor XII's effects on inflammation could overshadow the effects on coagulation. Schmaier thus wants to see additional studies control for levels of prekallikrein and Factor XI. Another way to answer the problem, he said, is to show that Factor XII activated by misfolded protein aggregates has higher specificity for prekallikrein than for Factor XI.

\section{Screening factor(y)}

Johan Renes, CEO of Crossbeta, said the nearterm applications of Factor XII are as a marker for misfolded proteins. He said measuring activated Factor XII in blood samples could help predict disease severity and progression in patients with protein-misfolding diseases.

Gebbink added that diagnostics and screening tools based on Factor XII also may be useful in detecting contamination in biopharmaceutical manufacturing. Misfolded proteins present in protein-based therapeutics can be immunogenic and lead to the production of antibodies that neutralize therapeutic activity.

Because the interaction between Factor XII and misfolded proteins is derived from a biological system, Gebbink thinks contamination screening tools based on Factor XII would be more sensitive than chemical-based screens.

Crossbeta holds multiple patents based on the interaction between Factor XII and misfolded proteins for use in developing diagnostics and therapeutics for misfolded-protein diseases. The company is not yet pursuing Factor XII as a therapeutic target.

\section{REFERENCES}

1. Maas, C. et al. J. Clin. Invest.; published online Aug. 21, 2008; doi: $10.1172 / \mathrm{JCl} 35424$ 
Contact: Martijn F.B.G. Gebbink, University Medical Center Utrecht, Utrecht, the Netherlands

e-mail: m.gebbink@umcutrecht.nl

2. Schmaier, A.H. Int. Immunopharmacol. 8,161-165 (2008)
COMPANIES AND INSTITUTIONS MENTIONED

Case Western Reserve University, Cleveland, Ohio

Crossbeta Biosciences B.V., Utrecht, the Netherlands

Dyax Corp. (NASDAQ:DYAX), Cambridge, Mass.

Temple University, Philadelphia, $\mathrm{Pa}$.

University Medical Center Utrecht, Utrecht, the Netherlands 